\title{
SITUACIÓN Y DETERMINANTES SOCIALES DE LA ANEMIA EN GESTANTES PERUANAS SEGÚN DISTRIBUCIÓN GEOGRÁFICA 2016-2017
}

\author{
Percy Minaya León 1,2a, Carlos A. Gonzales-Medina 1, 2, b, c, Félix Ayala Peralta 1, 2, b, d, \\ Augusto Elmer Racchumí Vela ${ }^{1, \mathrm{e}}$
}

\begin{abstract}
RESUMEN
Objetivos. Evaluar la situación y determinantes sociales de la anemia en gestantes de centros poblados y comunidades rurales del Perú durante el año 2017. Materiales y métodos. Estudio Transversal utilizando la base de datos de la Encuesta Demográfica y de Salud Familiar (ENDES) 2017. Se analizaron los registros de 8533 mujeres gestantes pertenecientes a centros poblados y comunidades rurales incluyendo gestantes que residen en la capital y capitales departamentales. Se analizó la estadística descriptiva y regresión logística. Resultados. El $28 \%$ de las pacientes presentaron anemia en la gestación. Aquellas pacientes que pertenecen a centros poblados y comunidades rurales del Perú tienen un OR 2,0 IC95\% (1,4-2,9) $p<0,001$ en comparación con las gestantes que residen en las capitales departamentales y la capital nacional. Conclusiones. Existe asociación entre el lugar de residencia geográfica de las gestantes en centros poblados y comunidades rurales con la presencia de anemia en el embarazo en la población peruana.
\end{abstract}

Palabras clave: Anemia; Gestación; Comunidad rural (Fuente: DeCS BIREME).

\section{STATUS AND SOCIAL DETERMINANTS OF ANAEMIA IN PERUVIAN PREGNANT WOMEN ACCORDING TO GEOGRAPHICAL DISTRIBUTION 2016-2017}

\begin{abstract}
Objectives. To evaluate the situation and social determinants of anemia in pregnant women in populated centers and rural communities in Peru during the year 2017. Materials and methods. Cross-sectional study using the Demographic and Family Health Survey (ENDES) 2017 database. The records of 8,533 pregnant women belonging to populated centres and rural communities were analysed, including pregnant women residing in the capital and departmental capitals. Descriptive statistics and logistic regression were analysed. Results. Twenty-eight per cent of the patients suffered from anaemia during pregnancy. Those patients who belong to populated centers and rural communities of Peru have an OR 2.0 IC95\% (1.4-2.9) $p<0.001$ compared to pregnant women who reside in the departmental capitals and the national capital. Conclusions. There is an association between the geographic residence of pregnant women in populated centers and rural communities with the presence of anemia in pregnancy in the Peruvian population.
\end{abstract}

Key words: Anemia; Gestation; Rural community (Source: MeSH NLM).

\section{INTRODUCCIÓN}

La anemia es una condición clínica que se caracteriza por la deficiencia cualitativa o cuantitativa de la hemoglobina o glóbulos rojos. Asimismo, produce una reducción del aporte de oxígeno a nivel sanguíneo y tisular ${ }^{1}$. La clasificación del nivel de anemia, según la Organización Mundial de la Salud, se basa en los valores de su concentración sérica. Con cifras de 10 a $10.9 \mathrm{mg} / \mathrm{dl}$ se considera anemia leve, de 9.9 a $7 \mathrm{mg} / \mathrm{dl}$, anemia moderada y menor de $7 \mathrm{mg} /$ $\mathrm{dl}$, anemia severa ${ }^{2}$. Es importante señalar que el CDC
(Centers for Disease Control and Prevention's) considera una definición distinta a la anterior, definiendo la anemia en el segundo trimestre menor de $10,5 \mathrm{mg} / \mathrm{dl}$ o una cifra de hematocrito menor de $32 \%{ }^{3}$. Esta última definición es avalada por el Colegio Americano de Ginecología y Obstetricia (ACOG) en sus distintos reportes y guías clínicas ${ }^{4}$.

Es importante, además, señalar que la anemia durante la gestación constituye un problema de salud pública en los países en vías de desarrollo dado que incrementa la

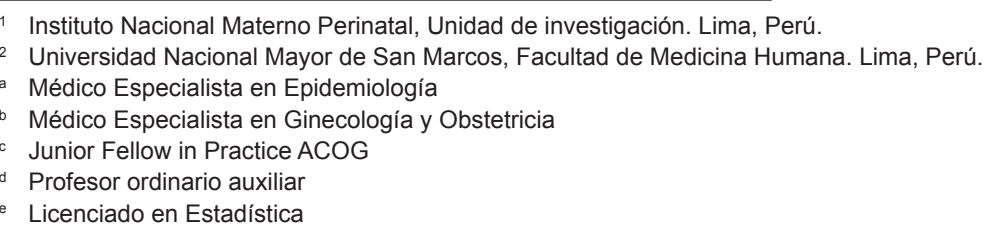

Citar como: Minaya P, Gonzales-Medina C, Ayala-Peralta F, Racchumi A. Situación y determinantes sociales de la anemia en gestantes peruanas según distribución geográfica 2016-2017. Rev Peru Investig Matern Perinat 2019; 8(1):23-9.

DOI: https://doi.org/10.33421/inmp.2019139

Recibido: 07-03-19 Aprobado: 27-03-19 
morbimortalidad materna y feto-neonatal ${ }^{5}$. Asimismo, la identificación oportuna de la anemia por deficiencia de hierro y el dosaje de ferritina son de vital importancia para un tratamiento óptimo ${ }^{6}$.

Un estudio multicentrico de cohortes, realizado en Nueva Zelandia, Irlanda e Inglaterra, determinó que existe un mayor riesgo de resultados perinatales adversos en mujeres que se hallan anémicas desde el principio de su embarazo. Por ejemplo, son más frecuentes los casos de bajo peso al nacer, APGAR menos de 7 y parto pretérmino. Sin embargo, estos resultados no son estadísticamente significativos ${ }^{7}$. Asimismo, el desarrollo mental en niños de zonas rurales se ve favorecido por la suplementación de hierro, ácido fólico y múltiples micronutrientes en China. Así lo demuestra un seguimiento realizado en los hijos de madres que recibieron suplementación con hierro prenatal que incluso no pudieron corregir a tiempo la anemia por deficiencia de hierro durante su embarazo ${ }^{8}$.

La tasa promedio global de anemia durante la gestación en el mundo es $43 \%$. Y en América Latina y el Caribe las tasas promedio es $37 \%$ de la población de gestantes de 15 a 49 años $^{9}$. Según, el último reporte nacional realizado el 2016 el Perú tiene una tasa de anemia en población gestante de $29 \%$.

El objetivo del presente estudio es evaluar la situación y determinantes sociales de la anemia en gestantes según su distribución geográfica en el Perú.

\section{MATERIALES Y MÉTODOS}

Estudio observacional sobre los casos incidentes de gestantes entrevistadas de las cuales se obtuvo datos de hemoglobina sanguínea, registrados en la Encuesta Demográfica y de Salud Familiar (ENDES-2017). Esta encuesta permite obtener información actualizada y analizar el cambio, tendencias y determinantes de la fecundidad, mortalidad y la salud en el Perú.

El diseño muestral de la encuesta ENDES es probabilístico, autoponderado por departamentos y de áreas, estratificado, multietápico e independiente para cada departamento. En cada departamento se seleccionan conglomerados y en cada conglomerado se seleccionan viviendas independientes a ser visitadas. Las poblaciones objetivo de la ENDES, se exploran mediante la aplicación de tres cuestionarios a) el cuestionario del hogar, que incluye el listado de miembros del hogar y las características de la vivienda; b) el cuestionario individual para MEF(mujeres en edad fértil) mujeres de 15 a 49 años de edad, que recoge datos de reproducción, anticoncepción, embarazo, parto, puerperio y lactancia, inmunización y salud, nupcialidad, preferencias de fecundidad, antecedentes del cónyuge y trabajo de la mujer, HIVISIDA y otras ITS, mortalidad materna, y violencia doméstica; c) el cuestionario de traumatismos y enfermedades crónicas, para todas las personas de 40 y más años de edad.

La determinación de hemoglobina en la ENDES se realiza mediante método colorimétrico con un equipo portátil HemoCue (HemoCue AB, Angelhome, Suecia). La precisión de Hemocue es bastante cercana a la obtenida por métodos directos con sangre venosa y arterial (El fundamento del método está basado en una reacción azidametahemoglobina, modificada del método de Vanzetti). El valor obtenido por el Hemocue en sangre capilar se corrige en base a la altitud en la que viven la población evaluada mediante la fórmula de Dirren et al.

La variable dependiente fue la existencia o no de anemia en las gestantes identificadas al momento de la encuesta, la anemia se confirmó cuando la hemoglobina sanguínea corregida tuvo un valor menor a $11 \mathrm{mg} / \mathrm{dL}$ Las variables independientes fueron agrupadas en tres grupos: a) variables sociodemográficas (área de residencia, altitud, región, quintil de bienestar al que pertenece el hogar, edad materna, grado de instrucción de madre, lengua materna de madre); c) variables relacionadas al cuidado materno e infantil (control prenatal, suplemento de hierro durante gestación, parto institucional). Todas las variables independientes se dicotomizaron para analizarse como categóricas.

Los criterios de inclusión incluyo a las mujeres gestantes al momento de la aplicación de la encuesta (pregunta 226 de la sección 2 del cuestionario individual, sobre reproducción) $\mathrm{y}$ tener el valor de hemoglobina registrado.

Se determinó la normalidad de los datos usando la prueba de Kolmogorov-Smirnov y en cifras menores de 30 se usó la prueba de Shapiro-Wilk. Asimismo, se determinó las frecuencias, medias y desviación estándar de las variables. Para la determinación de las diferencias de las variables cualitativas se usó la prueba Chi cuadrado y prueba exacta de Fisher para aquellas celdas con datos menores a cinco. Para el análisis bivariado se utilizó la odd ratio OR con sus respetivos intervalos de confianza al 95\% (IC 95\%) y la regresión logística binaria multivariante, bajo el método introducir. En todas las pruebas estadísticas se utilizó un $p$ menor 0,05 y se trabajó con el paquete estadístico SPSS 22.

La información fue utilizada de manera confidencial, la base de datos del ENDES no presenta identificador de algunos de los participantes. Además, como parte del trabajo de campo se solicita el consentimiento informado para la ejecución del estudio.

\section{RESULTADOS}

La población femenina del ENDES 2017 de 15 a 49 años es una población relativamente joven dado que el $45 \%$ son menores de 30 años de edad. Las participantes del estudio eran en su mayoría entre 20 y 29 años de edad. Asimismo, tenían educación escolar incluyendo primaria o secundaria. 
En la evaluación de las adolescentes gestantes se observó que los mayores porcentajes de ellas que alguna vez se embarazaron se encuentran en el área rural $(23,2 \%)$. Asimismo, las residentes de la Selva $(22,6 \%)$; y las que se encuentran en el quintil inferior de riqueza $(24,2 \%)$. En otro extremo, con menores porcentajes, están las adolescentes delárea urbana (10,7\%), las residentes en Lima Metropolitana $(9,7 \%)$ y las ubicadas en el quintil superior de riqueza $(3,9 \%)$. En la tabla 1 se describen las características de la población de estudio incluyendo, edad según grupos etarios, nivel de educación, lengua materna, lugar de residencia, inicio de controles prenatales y planificacion de la gestación actual.

El idioma materno se observó solo en el $18 \%$ de las pacientes que incluyen el quechua, aymara $u$ otra lengua nativa amerindia. La mayoría de las pacientes procedían de áreas urbanas en un $74 \%$ de las cuales alrededor de la mitad eran de la costa con igual porcentaje de Lima Metropolitana y el resto de la costa. El inicio de los controles prenatales se dio en el $26 \%$ durante el primer mes de gestación y $68 \%$ de las participantes no tenían hijos o solo tenían un hijo antes del embarazo actual. Cuando se entrevistó a las gestantes el $40 \%$ tenía una edad gestacional entre siete y nueve meses, y en el $61 \%$ de los casos el embarazo actual no estuvo planificado.

Cuando se realizó el análisis según la distribución geografica de las pacientes a nivel nacional se documentó según el estado de anemia durante la gestación actual (Figura 1). Se describe claramente que las gestantes con anemia severa se hallan geográficamente distribuidas

Tabla 1 Características sociodemográficas y prenatales de la población de estudio ENDES 2016-2017

\begin{tabular}{|c|c|c|c|c|c|c|c|c|}
\hline & & \multirow{2}{*}{\multicolumn{2}{|c|}{$\begin{array}{c}\text { Anemia } \\
\text { Embarazada con } \\
\text { anemia } \\
\end{array}$}} & \multirow{3}{*}{ Mediana } & \multicolumn{2}{|c|}{ Hemoglobina } & \multirow{3}{*}{ Chi2 } & \multirow{3}{*}{ p_value } \\
\hline & & & & & Percentil & Percentil & & \\
\hline & & $\mathrm{n}$ & $\%$ & & & & & \\
\hline \multirow{3}{*}{ Región Natural } & Costa & 84 & $35.9 \%$ & 10.30 & 9.70 & 10.60 & \multirow{3}{*}{.456} & \multirow{3}{*}{.796} \\
\hline & Sierra & 57 & $24.4 \%$ & 10.40 & 9.70 & 10.80 & & \\
\hline & Selva & 93 & $39.7 \%$ & 10.30 & 9.70 & 10.70 & & \\
\hline \multirow{4}{*}{$\begin{array}{l}\text { Edad actual } \\
\text { de la madre }\end{array}$} & $15-18$ & 7 & $3.0 \%$ & 9.90 & 9.30 & 10.80 & \multirow{4}{*}{3.011} & \multirow{4}{*}{.390} \\
\hline & $19-29$ & 143 & $61.1 \%$ & 10.30 & 9.90 & 10.60 & & \\
\hline & $30-39$ & 78 & $33.3 \%$ & 10.25 & 9.60 & 10.60 & & \\
\hline & $40-49$ & 6 & $2.6 \%$ & 9.65 & 9.10 & 10.50 & & \\
\hline \multirow{8}{*}{$\begin{array}{l}\text { Nivel de Altitud y } \\
\text { Región }\end{array}$} & Chala, menos de 500 msnm & 73 & $31.2 \%$ & 10.30 & 9.70 & 10.60 & \multirow{8}{*}{6.654} & \multirow{8}{*}{.354} \\
\hline & Yunga, de 501 a 2300 msnm & 22 & $9.4 \%$ & 10.50 & 10.10 & 10.80 & & \\
\hline & Quechua, de 2301 a 3500 msnm & 33 & $14.1 \%$ & 10.40 & 9.70 & 10.80 & & \\
\hline & Suni, de 3501 a 4000 msnm & 17 & $7.3 \%$ & 10.20 & 10.00 & 10.60 & & \\
\hline & Puna, de 4001 a 4800 msnm & 9 & $3.8 \%$ & 10.00 & 8.90 & 10.30 & & \\
\hline & Janca, de 4801 a 6768 msnm & 0 & $0.0 \%$ & & & & & \\
\hline & Selva baja, de 80 a 400 msnm & 59 & $25.2 \%$ & 10.30 & 9.70 & 10.70 & & \\
\hline & Selva alta, de 401 a 1000 msnm & 21 & $9.0 \%$ & 10.20 & 9.90 & 10.70 & & \\
\hline \multirow{2}{*}{$\begin{array}{l}\text { Tipo de lugar de } \\
\text { residencia }\end{array}$} & Rural & 82 & $35.0 \%$ & 10.40 & 9.90 & 10.70 & \multirow{2}{*}{0.298} & \multirow{2}{*}{.585} \\
\hline & Urbano & 152 & $65.0 \%$ & 10.30 & 9.70 & 10.60 & & \\
\hline \multirow{4}{*}{ Nivel de Urbanización } & $\begin{array}{l}\text { Capital nacional - Lima } \\
\text { Metropolitana }\end{array}$ & 27 & $11.5 \%$ & 10.30 & 9.20 & 10.60 & \multirow{4}{*}{1.458} & \multirow{4}{*}{.692} \\
\hline & $\begin{array}{l}\text { Capitales departamentales y } \\
\text { cuidades grandes }\end{array}$ & 66 & $28.2 \%$ & 10.30 & 9.80 & 10.70 & & \\
\hline & $\begin{array}{l}\text { Pequeños Centros Poblados } \\
\text { Urbanos }\end{array}$ & 59 & $25.2 \%$ & 10.20 & 9.60 & 10.60 & & \\
\hline & Comunidades Rurales & 82 & $35.0 \%$ & 10.40 & 9.90 & 10.70 & & \\
\hline \multirow{5}{*}{ Quintil de riqueza } & Quintil inferior & 77 & $32.9 \%$ & 10.40 & 9.90 & 10.70 & \multirow{5}{*}{4.527} & \multirow{5}{*}{.339} \\
\hline & Segundo quintil & 68 & $29.1 \%$ & 10.20 & 9.50 & 10.60 & & \\
\hline & Quintil intermedio & 42 & $17.9 \%$ & 10.30 & 9.70 & 10.60 & & \\
\hline & Cuarto quintil & 30 & $12.8 \%$ & 10.30 & 9.90 & 10.50 & & \\
\hline & Quintil superior & 17 & $7.3 \%$ & 10.60 & 9.80 & 10.80 & & \\
\hline \multirow{2}{*}{$\begin{array}{l}\text { Etnia por idioma } \\
\text { aprendido en la niñez }\end{array}$} & Etnia & 51 & $21.8 \%$ & 10.40 & 10.20 & 10.70 & \multirow{2}{*}{3.270} & \multirow{2}{*}{.071} \\
\hline & Castellano o lengua extranjera & 183 & $78.2 \%$ & 10.30 & 9.70 & 10.60 & & \\
\hline
\end{tabular}


Tabla 2. Características sociodemográficas y prenatales de la población de estudio ENDES 2016-2017

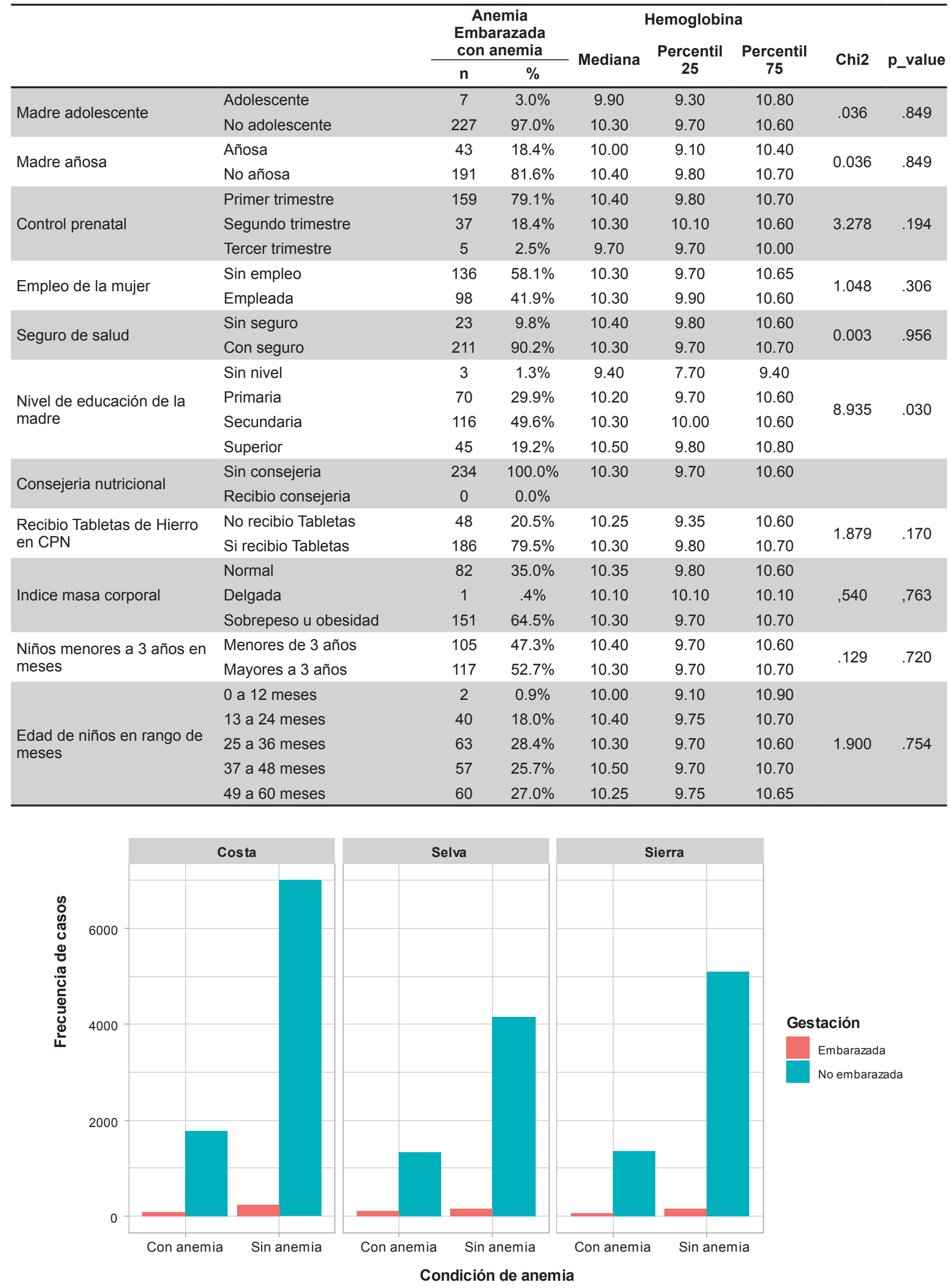

Figura 1. Distribución de la condición de anemia en mujeres peruanas según zona geográfica a nivel nacional 
principalmente en la selva. Asimismo, no existen casos de anemia severa en la sierra según los reportes actuales. Además, la anemia leve constituye el tipo más frecuente en todas las regiones.

Cuando se realizó el análisis bivariado de los factores potencialmente asociados a la anemia en la gestación se determinó que el lugar de residencia rural y pertenecer a la ubicación geográficas de pequeños centros poblados y comunidades rurales está fuertemente asociado a la presencia de anemia en la gestación.
En tabla 2 se describen los factores asociados a la condición de anemia en la gestación actual los cuales los clasificamos en los siguientes grupos:

a) Sociodemográficos: incluyen el lugar de residencia rural, la ubicación geográfica de pequeños centros poblados y comunidades rurales. Además, pertenecer a quintiles inferiores Q1 y Q2;

b) Culturales: presencia de analfabetismo funcional, la exposición a medios de comunicación, el nivel de

Tabla 3. Factores asociados a la condición de gestante y la presencia de anemia. ENDES 2017

\begin{tabular}{|c|c|c|c|c|c|c|c|c|}
\hline & \multirow[b]{2}{*}{ Variable } & \multirow{2}{*}{$\begin{array}{c}\text { Gestante } \\
\text { con } \\
\text { anemia }\end{array}$} & \multirow{2}{*}{$\begin{array}{c}\text { Gestante } \\
\text { sin } \\
\text { anemia }\end{array}$} & \multirow{2}{*}{ OR } & \multicolumn{2}{|c|}{ IC $95 \%$} & \multirow{2}{*}{$\begin{array}{l}\text { Chi } \\
\mathrm{X} 2\end{array}$} & \multirow[b]{2}{*}{ p-valor } \\
\hline & & & & & $\begin{array}{l}\text { Límite } \\
\text { inferior }\end{array}$ & $\begin{array}{l}\text { Límite } \\
\text { superior }\end{array}$ & & \\
\hline \multirow{2}{*}{ Lugar de residencia } & Rural & 64 & 2173 & \multirow{2}{*}{2,4} & \multirow{2}{*}{1,7} & \multirow{2}{*}{3,4} & \multirow{2}{*}{27,9} & \multirow{2}{*}{$<0,001$} \\
\hline & Urbano & 76 & 6221 & & & & & \\
\hline \multirow{2}{*}{ Ubicación geografica } & $\begin{array}{l}\text { Pequeños centros poblados y } \\
\text { comunidades rurales }\end{array}$ & 93 & 4168 & \multirow[t]{2}{*}{2,0} & \multirow[t]{2}{*}{1,4} & \multirow[t]{2}{*}{2,9} & \multirow[t]{2}{*}{15,5} & \multirow{2}{*}{$<0,001$} \\
\hline & Capital Nacional o departamentales & 47 & 4225 & & & & & \\
\hline \multirow{2}{*}{ Quintil socioeconomico } & Quintiles inferiores Q1 y Q2 & 93 & 3949 & \multirow{2}{*}{2,2} & \multirow{2}{*}{1,6} & \multirow{2}{*}{3,2} & \multirow{2}{*}{20,7} & \multirow{2}{*}{$<0,001$} \\
\hline & Quintiles No pobres & 47 & 4445 & & & & & \\
\hline \multirow{2}{*}{ Analfabetismo Funcional } & No lee o lee con dificultad & 22 & 464 & \multirow{2}{*}{3,2} & 20 & 51 & 270 & $<0001$ \\
\hline & Lee facilmente & 117 & 7930 & & 2,0 & 0,1 & $2 r, 0$ & $<0,001$ \\
\hline Nivel de exposición a & Una vez por semana o menos & 126 & 6433 & 2,9 & 1,6 & 5,1 & 14,5 & $<0,001$ \\
\hline medios & Todos los dias & 13 & 1926 & & & & & \\
\hline Grado de inctrurción & Primaria o sin estudio & 56 & 1728 & 2,6 & 1,8 & 3,6 & 31,4 & $<0,001$ \\
\hline Grado de instruccion & Secundaria o superior & 84 & 6666 & & & & & \\
\hline Nivel de autonomía en el & No toma decisiones & 28 & 842 & 2,0 & 1,3 & 3,1 & 11,0 & $<0,001$ \\
\hline cuidado de su salud & Sola o en conjunto con su pareja & 104 & 6329 & & & & & \\
\hline Situacinn laboral & Sin empleo & 76 & 3593 & 1,6 & 1,1 & 2,2 & 7,4 & 0,008 \\
\hline situacion ravoral & Empleada & 64 & 4801 & & & & & \\
\hline Controles nrenatales & Menor de 6 controles & 25 & 732 & 2,4 & 1,5 & 3,8 & 16,2 & $<0,001$ \\
\hline Comitries prenatales & Mayor o igual a 6 controles & 92 & 6576 & & & & & \\
\hline Percona gu realiza el CPN & Empirico & 5 & 78 & 4,4 & 1,8 & 11,1 & 11,8 & 0,001 \\
\hline Persona que realiza el crIv & Profesional & 104 & 7161 & & & & & \\
\hline Necesidades de & $\mathrm{SI}$ & 46 & 426 & 8,5 & 5,8 & 12,2 & 179,2 & $<0,001$ \\
\hline planificacion insatisfechas & No & 86 & 6745 & & & & & \\
\hline Parto inctitucionalizado & No & 40 & 609 & 5,1 & 3,5 & 7,4 & 88,5 & $<0,001$ \\
\hline Parto institucionalizauo & $\mathrm{Si}$ & 100 & 7753 & & & & & \\
\hline Años de educación & Menor o igual a 8 años & 68 & 2317 & 2,5 & 1,8 & 3,5 & 30,0 & $<0,001$ \\
\hline 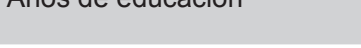 & Mas de 9 años & 72 & 6077 & & & & & \\
\hline Tipo de parto en gestación & Cesarea & 113 & 5475 & 2,2 & 1,5 & 3,4 & 14,6 & $<0,001$ \\
\hline anterior & Vaginal & 27 & 2918 & & & & & \\
\hline Tino de cecarea anterior & Emergencia & 10 & 1490 & 1,6 & 1,5 & 3,4 & 14,6 & $<0,001$ \\
\hline IIpu ne cesarea amterion & Electiva & 17 & 1428 & & & & & \\
\hline Prescripción de Hierro oral & $\mathrm{Si}$ & 36 & 1576 & 1,4 & 1,0 & 2,2 & 4,3 & 0,037 \\
\hline en CPN & No & 104 & 6818 & & & & & \\
\hline Idioma (aprendido desde la & Etnico & 37 & 1349 & 1,9 & 1,3 & 2,7 & 10,5 & 0,001 \\
\hline niñez) & Castellano o extranjero & 103 & 7044 & & & & & \\
\hline
\end{tabular}

OR: Odds Ratio, análisis bivariado; IC: Intervalo de confianza al 95\%; Chi X2: Prueba Chi cuadrado; $p$-valor: Nivel de significancia estadística menor de 0,05 
instrucción primaria o sin estudio, menos de 8 años de educación, idioma étnico aprendido desde la niñez, necesidades de planificación insatisfechas y pobre nivel de autonomía en la toma de decisiones del cuidado de su salud.

c) Condiciones obstétricas: menos de 6 controles prenatales, persona empírica que realiza el control prenatal, parto no institucionalizado, historia de cesárea previa, antecedente de cesárea de emergencia en gestación previa y no prescripción de hierro oral durante el control prenatal.

En el análisis de los factores potenciales asociados a la condición de anemia en gestantes, se halló que no existe asociación estadística entre la edad materna avanzada $(p=0,75)$, bajo índice de masa corporal $(p=0,17)$ y la talla materna menor de 140 centímetros $(p=0,67)$ con la condición de anemia en la gestación.

\section{DISCUSIÓN}

En Estados Unidos la prevalencia de anemia en mujeres en edad fértil es 21,55 por 1000 mujeres cuando la anemia se definió con una hemoglobina menor de $10 \mathrm{~g} /$ $\mathrm{dl}^{10}$. Asimismo, en dicha población existe mayor riesgo de anemia en la gestación en la población de raza negra, incrementándose dos veces el riesgo en la población blanca no hispana ${ }^{10}$.

Un estudio peruano que evaluó los niveles de hemoglobina en gestantes adolecentes a nivel nacional demostró que los niveles de hemoglobina son en promedio menores en zonas alto andinas ${ }^{11}$.

En una revisión sistemática reciente se demuestra que la suplementación de hierro a las gestantes con anemia por deficiencia de hierro no afecta el estado de hierro infantil en los niños menores de 6 meses ${ }^{12}$.

Se ha demostrado que las concentraciones bajas de hemoglobina materna antes o después del embarazo incrementan el riesgo de anemia en los recién nacidos de 3 a 5 años. Esta afirmación se registró durante el seguimiento de 78923 mujeres con embarazo único con datos de la hemoglobina previa a la gestación, durante el primer control prenatal, el segundo y tercer trimestre. Asimismo, se halló el nivel de hemoglobina de sus hijos a los 3 y 5 años encontrando una asociación entre los niveles de hemoglobina maternos y la de sus hijos ${ }^{13}$.

En un estudio transversal peruano realizado el 2012 cuyo objetivo fue evaluar las prácticas del cuidado maternoinfantil en las áreas con pobreza extrema determinó que el $69 \%$ de las madres entrevistadas hicieron su primer control prenatal en el primer trimestre. $Y$ el $65,3 \%$ de ellas cumplieron más de 6 controles prenatales. Asimismo, la práctica de la suplementación con hierro a sus hijos se halló en solo $30 \%$ de las madres posterior a la lactancia materna exclusiva y se calificó por los autores como baja frecuencia ${ }^{14}$.

Durante el embarazo existe un requerimiento de hierro mayor debido a la expansión del volumen sanguíneo aproximadamente que se da alrededor del $50 \%(1000 \mathrm{~mL})$ y un aumento de la masa de eritrocitos alrededor del $25 \%$ $(300 \mathrm{~mL})$. Estos datos son registrados en un embarazo único ${ }^{15}$.

Sin embargo, la información exacta de la prevalencia por deficiencia de hierro en la población gestante resulta limitada, por la forma de recolectar o el sub registro de la informaciónv ${ }^{16}$.

Nuestros hallazgos concuerdan con lo señalado el 2015 sobre el análisis espacial a nivel nacional de la distribución de la anemia gestacional en nuestro país. En este documento se señala que la mayor distribución de esta entidad se sitúa en el área rural y sur de la sierra. Asimismo, los conglomerados de con altas prevalencias de anemia gestacional coinciden con las zonas de alta prevalencia regional ${ }^{17}$.

Financiamiento: Autofinanciado.

Conflicto de interés: Los autores declaran no tener algún conflicto de intereses.

\section{REFERENCIAS BIBLIOGRÁFICAS}

1. Grewal A. Anaemia and pregnancy: Anaesthetic implications. Indian J Anaesth. 9 de enero de 2010;54(5):380.

2. Organización Mundial de la Salud. Concentraciones de hemoglobina para diagnosticar la anemia y evaluar su gravedad. Haemoglobin concentrations for the diagnosis of anaemia and assessment of severity [Internet]. 2011 [citado 8 de agosto de 2018]; Disponible en: http://apps.who.int/iris/handle/10665/85842

3. Recommendations to prevent and control iron deficiency in the United States. Centers for Disease Control and Prevention. MMWR Recomm Rep Morb Mortal Wkly Rep Recomm Rep. 3 de abril de 1998;47(RR-3):1-29.

4. American College of Obstetricians and Gynecologists. ACOG Practice Bulletin No. 95: anemia in pregnancy. Obstet Gynecol. julio de 2008;112(1):201-7.

5. WHO. The global prevalence of anaemia in 2011 [Internet]. WHO. [citado 20 de agosto de 2018]. Disponible en: http:// www.who.int/entity/nutrition/publications/micronutrients/global_prevalence_anaemia_2011/en/index.html

6. Lazarte S, Issé B. Prevalencia y etiología de anemia en el embarazo. Estudio observacional descriptivo en el Instituto de Maternidad de Tucumán. Rev Argent Salud Pública. 1 de enero de 2011;2:28.

7. Masukume G, Khashan AS, Kenny LC, Baker PN, Nelson G. Risk Factors and Birth Outcomes of Anaemia in Early Pregnancy in a Nulliparous Cohort. PLoS ONE [Internet]. 15 de abril de 2015 [citado 8 de agosto de 2018];10(4). Disponible en: https://www.ncbi.nlm.nih.gov/pmc/articles/PMC4398319/ 
8. Chang S, Zeng L, Brouwer ID, Kok FJ, Yan H. Effect of iron deficiency anemia in pregnancy on child mental development in rural China. Pediatrics. marzo de 2013;131(3):e755-763.

9. Stevens GA, Finucane MM, De-Regil LM, Paciorek CJ, Flaxman SR, Branca F, et al. Global, regional, and national trends in haemoglobin concentration and prevalence of total and severe anaemia in children and pregnant and non-pregnant women for 1995-2011: a systematic analysis of population-representative data. Lancet Glob Health. 1 de julio de 2013;1(1):e16-25.

10. Adebisi OY, Strayhorn G. Anemia in pregnancy and race in the United States: blacks at risk. Fam Med. octubre de 2005;37(9):655-62.

11. Munares-García O, Gómez-Guizado G. Niveles de hemoglobina y anemia en gestantes adolescentes atendidas en establecimientos del Ministerio de Salud del Perú, 2009-2012. Rev Peru Med Exp Salud Pública [Internet]. 25 de septiembre de 2014 [citado 20 de agosto de 2018];31(3). Disponible en: http://www.rpmesp.ins.gob.pe/index.php/rpmesp/article/ view/87

12. Cantor AG, Bougatsos C, Dana T, Blazina I, McDonagh M. Routine iron supplementation and screening for iron deficiency anemia in pregnancy: A systematic review for the U.S. preventive services task force. Ann Intern Med. 21 de abril de 2015;162(8):566-76.

13. Zhang Y, Jin L, Liu J-M, Ye R, Ren A. Maternal haemoglobin concentrations before and during pregnancy as determinants of the concentrations of children at 3-5 years of age: A large follow-up study. Eur J Clin Nutr. 17 de agosto de 2018;

14. Hurtado V, Enrique J, Solís Alcedo L, Valdez V, Eduardo W, Aguirre $\mathrm{R}$, et al. Evaluación de las prácticas de cuidado materno infantil en áreas con pobreza extrema del Perú, 2012. Rev Peru Med Exp Salud Pública. 2014;31:243-53.

15. Pitkin RM. Nutritional influences during pregnancy. Med Clin North Am. 1977;61(1):3-15.

16. Oregon Evidence-based Practice Center. Screening for Iron Deficiency Anemia in Childhood and Pregnancy: Update of the 1996 U.S. Preventive Task Force Review [Internet]. Rockville (MD): Agency for Healthcare Research and Quality (US); 2006 [citado 20 de agosto de 2018]. (U.S. Preventive Services Task Force Evidence Syntheses, formerly Systematic Evidence Reviews). Disponible en: http://www.ncbi.nlm.nih. gov/books/NBK33399/

17. Hernández-Vásquez $A$, Azañedo $D$, Antiporta DA, Cortés $S$. Análisis espacial de la anemia gestacional en el Perú, 2015. Rev Peru Med Exp Salud Pública. 2017;34(1):43-51.

Correspondencia:

Dirección: Edificio Los Caobos, Depto. 1401, Residencial San felipe, Jesús María,Lima.

Correo electrónico: percyminaya@gmail.com

Teléfono: 934739439 